\title{
Infant Vaccinations among Mothers with Substance-Use Disorders: A Comparative Study
}

\author{
Ann C. Falkenberg-Olson, PhD; Karen L. Hayter, PhD; Renee A. Holzer, BSN; \\ Jayne M. Laylan, MSN; Andrew J. Borgert, PhD; and Ann E. Budzak Garza, MD
}

\begin{abstract}
Introduction: Infants of mothers with substance use disorder (SUD) are exposed to complex social environments and increased childhood health risks that can lead to adverse consequences throughout the lifespan. GunderKids, a voluntary, specialized, comprehensive pediatric care management program, was developed to mitigate many of these adverse consequences. Our organization is evaluating several clinical outcomes related to health and development in children born to women with SUD. The current study addressed the timeliness of vaccination coverage among these infants.
\end{abstract}

Methods: This descriptive comparative preliminary study evaluated data of infants and their mothers with SUD who were previously identified during prenatal care visits either by self-report or by positive urine screens. Sociodemographic and vaccination data were extracted from a longitudinal master dataset of variables developed and maintained through retrospective review of electronic health records (EHRs) of these mothers and their infants. Timeliness of vaccination coverage of SUD-exposed infants participating in GunderKids was compared with that of SUD-exposed infants receiving standard pediatric care and was determined using a cumulative vaccinations method.

Results: Overall, infants in the GunderKids group $(n=50)$ had more timely vaccination coverage than those receiving standard pediatric care $(n=20)$. Examples of timelier coverage included Haemophilus influenzae type b (Hib) at 4 months $(P=.0$ I; OR $4.3,95 \% \mathrm{Cl}$ I.4-13.4), for pneumococcal $(P=.004$; OR 6.6, 95\% Cl I.8-23.8) and $\mathrm{Hib}(P=.004 ; \mathrm{OR} 5.8,95 \% \mathrm{Cl}$ I.6-2I.9) vaccinations at 15 months. More than $77 \%$ of GunderKids received all 6-month vaccinations in a timely manner compared with less than $45 \%$ of the standard care group; odds ratios suggest that GunderKids had 4.0-5.6 higher odds of receiving 6-month vaccinations.

Conclusion: Vaccination coverage of infants participating in GunderKids was timelier than that of infants receiving standard pediatric care. Results suggest that specialized programs like GunderKids may assist in mitigating adverse health consequences and timeliness of vaccination coverage might be used as a proxy for measuring program effectiveness. Further investigation is recommended to determine clinical, individual, and organizational factors that influence parental behaviors and pediatric outcomes within SUD-exposed families.

Keywords: Timely infant vaccination coverage; Substance use disorders; Pediatric care management

Corresponding Author: Karen L. Hayter, PhD, Gundersen Health System, 1836 South Avenue, La Crosse,WI 5460I, Email: klhayter@gundersenhealth.org
Received: May 8, 2020

Revised: July 14, 2020

Accepted: August 15, 2020

doi: $10.3|2| / \mathrm{cmr} .2020 .1580$ 
$\mathrm{T}$ The United States is experiencing a crisis related to substance use disorder (SUD) and its long-term consequences. ${ }^{1}$ A regional study conducted in our Midwestern health system found that the rate of SUD among pregnant women was higher than anticipated. ${ }^{2}$ Infants born to mothers with SUD are likely to be exposed to complex social environments and increased childhood health risks that can have lifelong adverse consequences. Inadequate medical and dental care, among other health risks, ${ }^{3,4}$ are globally recognized among children whose parents misuse drugs and alcohol., ${ }^{5,6}$ For example, nearly $25 \%$ of Australian mothers who use illicit substances are not accessing standard child health services during their child's first 2 years of life. ${ }^{7}$ Mothers with SUD may not keep timely well-child appointments owing to their fear of staff referrals to child protective services and judgmental attitudes of healthcare staff. ${ }^{8}$

Evidence supports that integrated clinical pediatric care combining well-child services with addiction services demonstrates better clinical outcomes in child development and growth in SUD-exposed children than do standard clinical pediatric services that are not integrated. ${ }^{9}$ Literature also supports that parents are more likely to have their children vaccinated when clinicians integrate an honest nonconfrontational manner in approaching childhood vaccinations ${ }^{10}$ and if provider competence is trusted. ${ }^{11}$ Ageappropriate timely vaccinations during infancy lead to decreased individual risk in contracting vaccine-preventable diseases, reduced risk for large community-acquired outbreaks $^{12}$ and to significant decreased direct and indirect costs related to having to revaccinate. ${ }^{13}$

In response to a growing number of pregnant women with SUD, our Midwestern healthcare organization developed GunderKids, a specialized pediatric care management program for children of mothers with current diagnosis or history of SUD. ${ }^{8}$ A key focus of the GunderKids program is to develop and maintain respectful caring relationships between GunderKids families and staff. ${ }^{8}$ The program provides integrated multidisciplinary care, individualized assessments, education, vaccinations, role modeling, support, and timely referrals based on participants' needs.

The overall success of programs like GunderKids is difficult to determine and is largely based on anecdotal or qualitative evidence; however, we suggest that quantifiable healthcare milestones might be used as proxy to measure program effectiveness. Therefore, in the current study, we compared timely vaccination coverage of SUD-exposed infants participating in GunderKids with that of SUD-exposed infants receiving standard pediatric care.

\section{Methods}

This Institutional Review Board approved retrospective study was conducted in a Midwestern healthcare system that serves southwestern Wisconsin, southeastern Minnesota, and northeastern Iowa. The system includes a 325-bed tertiary care hospital, 6 critical access hospitals, and 30 clinics.

Infants born between December 1, 2015, and March 13, 2017 to mothers aged 18 years or older with SUD were included. These mothers were identified as having SUD during prenatal care visits either by self-report or by urine screens positive for illicit drugs. ${ }^{8}$ A patient referral system was established that included referrals from the high-risk obstetrics or family medicine teams to GunderKids nurses who met with the expectant parent(s) during a prenatal appointment, while in-patient postpartum, or at the first newborn pediatric visit to discuss the program and offer information. All potential participants were offered the opportunity to enroll in GunderKids for their infant's pediatric care.

Infants were therefore divided into two groups: those whose mothers voluntarily enrolled in GunderKids and attended at least one clinic appointment with GK staff $(\mathrm{n}=50)$, and those whose mothers did not participate in GunderKids and contemporaneously received standard pediatric care $(n=20)$. Sociodemographic and vaccination data were used to create a subset from the longitudinal master dataset of variables developed and maintained through retrospective review of electronic health records (EHRs). Two members of the research staff extracted all vaccination data through December 31, 2019. Interrater reliability was performed by these members and included at least $5 \%$ of all participant data; discrepant data were reviewed and corrected by consensus.

\section{Definitions of Terms}

Several terms explain key components in this comparative analysis and are discussed further.

Study follow-up is defined as the time (in months) that an infant received clinical care as the first recorded clinic pediatric visit after birth to the last recorded contact date within our health system or the last recorded vaccination date.

Eligible infants refers to infants who met age requirements for a specific vaccination, received all prior up to date vaccinations, and were due for that specific vaccination.

Up to date refers to an infant receiving all required number of doses for a specific vaccination by a certain age in months. For example, infants were considered up to date for diphtheria, tetanus, and pertussis (DTaP) at 12 months if they had 3 total doses of DTaP by the age of 12 months including a grace period of 30 days. Infants who were not up to date at a given timepoint could receive additional doses to allow catch up with the recommended vaccination schedule.

Grace period allows for a specific amount of time for an infant to receive a vaccination after the recommended age-related date. A 30-day grace period was added to the end of each vaccination age-recommended due date, except for the birth dose of hepatitis B (Hep B) which followed Centers for Disease Control 
guidelines $^{14}$ recommending vaccination prior to hospital discharge.

Timeliness of vaccination coverage is defined in terms of a ratio and was calculated by dividing the number of eligible infants who received a specific vaccination, by the number of eligible infants who were due to receive that vaccination at a given timepoint, inclusive of grace period. When summarizing overall timeliness of a specific vaccination at any given timepoint, infants whose study follow-up ended prior to the end of the 30-day grace period were not included in the analysis for that timepoint unless they had already received a specific up to date vaccine. For example, four doses of the pneumococcal vaccine (PCV) and Haemophilus influenzae type b (Hib) vaccines are recommended by age 15 months. An infant whose follow-up ended at age 15 months and 1 day who received all four recommended doses of $\mathrm{PCV}$ would be included in the 15 -month analysis for PCV. That same infant who received only 3 of 4 doses of Hib would not be included in the 15-month analysis for Hib because that infant's study follow-up did not extend beyond the 30-day grace period. Vaccinations that allowed for a range of time to receive a specific dose were evaluated at the end of the recommended time range. For example, the third doses of Hep B and polio were due between 6 and 18 months of age. Therefore, third dose timeliness of vaccination coverage for both vaccinations was calculated at the end of the range or 18 months.

\section{Study Protocol}

Vaccinations included in the analysis were hepatitis B (Hep $\mathrm{B})$, diphtheria, tetanus, and pertussis (DTaP), pneumococcal
(PCV), Haemophilus influenzae type b (Hib), polio, rotavirus, measles/mumps/rubella (MMR), hepatitis A (Hep A) and varicella. Vaccination data for annual influenza were not obtained because of potential incomplete EHR data. Combination vaccinations were entered into the dataset as single vaccinations using the date the combination was administered. Timeliness of vaccination coverage for infants from birth to 24 months was compared between the GunderKids and standard pediatric care groups, and was analyzed at birth and at 2, 4, 6, 15, 18, and 23 months. Timeliness of vaccination coverage was determined using a cumulative vaccination dose method where, based on guidelines from the Centers for Disease Control, ${ }^{12}$ infants were required to receive a certain number of doses of a vaccine by a certain age in order to be considered up to date for that vaccine.

Comparison of sociodemographic and clinical features, and vaccination coverage timeliness between groups utilized the chisquared and Fisher's exact tests for categorical data, and the Wilcoxon rank sum test for ordinal and continuous data. A $P$ value of $<.05$ was considered significant. Haldane-Anscombe corrected odds ratios were reported with Wald modified confidence intervals. All analyses were performed using the SAS software suite, version 9.4 (SAS Foundation, Cary NC).

\section{Results}

Sociodemographic characteristics of mothers of both groups are provided in Table 1. Most characteristics for mothers of GunderKids and those choosing standard pediatric care were similar. However, GunderKids mothers were more likely to be single or living with a partner who was not the father of the baby

Table 1. Sociodemographic Characteristics of Mothers with SUD per Group

\begin{tabular}{|c|c|c|c|}
\hline Characteristic & $\begin{array}{l}\text { GunderKids } \\
\qquad \mathrm{n}=50\end{array}$ & $\begin{array}{l}\text { Standard Pediatric Care } \\
\qquad \mathrm{n}=20\end{array}$ & $\boldsymbol{P}$ \\
\hline Mean Age at delivery (range), years & $27.2(18-41)$ & $28.8(22-37)$ & .13 \\
\hline Years of education (range)* & $12.3(10-16)$ & $12.4(10-16)$ & .73 \\
\hline Race, n (\%)† & & & .50 \\
\hline White & $41(84)$ & $18(90)$ & \\
\hline Other & $8(16)$ & $2(10)$ & \\
\hline Ethnicity, n (\%) & & & .36 \\
\hline Hispanic & $2(4)$ & $0(0)$ & \\
\hline Non-Hispanic & $48(96)$ & $20(100)$ & \\
\hline Employed, n (\%)‡ & $16(33)$ & 7 (39) & .63 \\
\hline Living arrangement, n (\%) & & & $<.001$ \\
\hline Not Partnered & $29(58)$ & $2(10)$ & \\
\hline With Partner, not FOB & $3(6)$ & $1(5)$ & \\
\hline With Partner, FOB & $18(36)$ & $17(85)$ & \\
\hline
\end{tabular}

FOB, father of the baby.

*Data for 47 GunderKids mothers (not available for 3 mothers).

†Data for 49 GunderKids mothers (not available for 1 mother).

fData for 49 GunderKids mothers and 18 Standard Pediatric Care mothers (not available for 1 and 2 mothers, respectively). 
than were mothers choosing standard pediatric care $(P=.03)$. Of note, study follow-up (in months) was longer for GunderKids (mean 28.5, SD 11.6; range 3.5-46.3) than for standard care infants (mean 21.8, SD 10.3; range 0.7-41.7) $(P=.03)$. Figure 1 graphically compares percentages of timely vaccination coverage for both groups according to age. Age-specific vaccination coverage data are compared in Table 2. For example, 48 of the 50 GunderKids infants were eligible for the third PCV dose at 6 months, with $38(79.2 \%)$ of the 48 receiving the vaccination. In contrast, 18 of the 20 standard pediatric care infants were eligible for the third PCV dose at 6 months, with 8 (44.4\%) of the 18 receiving the vaccination. At 6 months of age, GunderKids infants received significantly timelier PCV vaccinations than infants receiving standard pediatric care $(P=.006$; OR $4.5,95 \%$ CI 1.5-14.1) suggesting that GunderKids had 4.5 times higher odds of timelier PCV vaccination at 6 months. The odds ratio of 4.5 with a confidence interval of 1.5 to 14.1 suggests that there is a $95 \%$ probability that the true odds ratio would be likely to lie in the range 1.5-14.1 assuming no bias or confounding factors. Table 2 data are discussed in the following text:

Birth. All infants in both groups received timely Hep B vaccinations at birth, with all receiving recommended Hep B prior to discharge.

Two months. The overall percentages of timely vaccination coverage decreased slightly in both groups. No significant differences between groups were found.

Four months. Overall timely vaccination coverage continued to decrease in both groups; however, approximately $80 \%$ of GunderKids received all 4-month vaccinations in a timely manner. Over $61 \%$ of standard pediatric care infants received
DTaP, PCV, polio, and rotavirus vaccines on time, but the GunderKids group had a significantly higher percentage of timely coverage for the second dose of Hib than the standard care group ( $81.6 \%$ vs $50 \%$, respectively; $P=.01$; OR $4.3,95 \%$ CI 1.4-13.4).

Six months. The percentage of timely vaccination coverage plateaued for GunderKids infants at 6 months; however, all vaccinations were timelier in the GunderKids group. Only $44.4 \%$ of the standard care group received their third-dose rotavirus and pneumococcal vaccines, compared with $79.2 \%$ of GunderKids infants $(P=.006$; OR 4.5, 95\% CI 1.5-14.1). GunderKids vaccinations were also timelier for third-dose Hib vaccinations than those for standard care infants $(P=.002$; OR $5.6,95 \%$ CI 1.8-17.7). More than 77\% of GunderKids received all 6-month vaccinations in a timely fashion compared with less than $45 \%$ of the standard care group with odd ratios suggesting that GunderKids had 4.0-5.6 times higher odds of timelier 6-month vaccinations.

Fifteen months. Timely vaccination coverage for Hib continued to decrease for both the GunderKids $(60.5 \%)$ and the standard pediatric care (18.8\%) groups, with GunderKids receiving more timely vaccinations $(P=.004$; OR 5.8, 95\% CI 1.6-21.9). Pneumococcal vaccination coverage was timelier for GunderKids infants $(88.9 \%)$ than for standard care infants $(52.9 \%)(P=.004$; OR 6.6, 95\% CI 1.8-23.8). No differences between groups in timely vaccination coverage for $\operatorname{MMR}(P=.26)$ or varicella $(P$ $=.16)$ were found.

Eighteen months. No significant differences between groups were found in timely vaccination coverage for third-dose Hep B $(P=.99)$, fourth dose DTaP $(P=.08)$ or polio $(P=.55)$. Of the

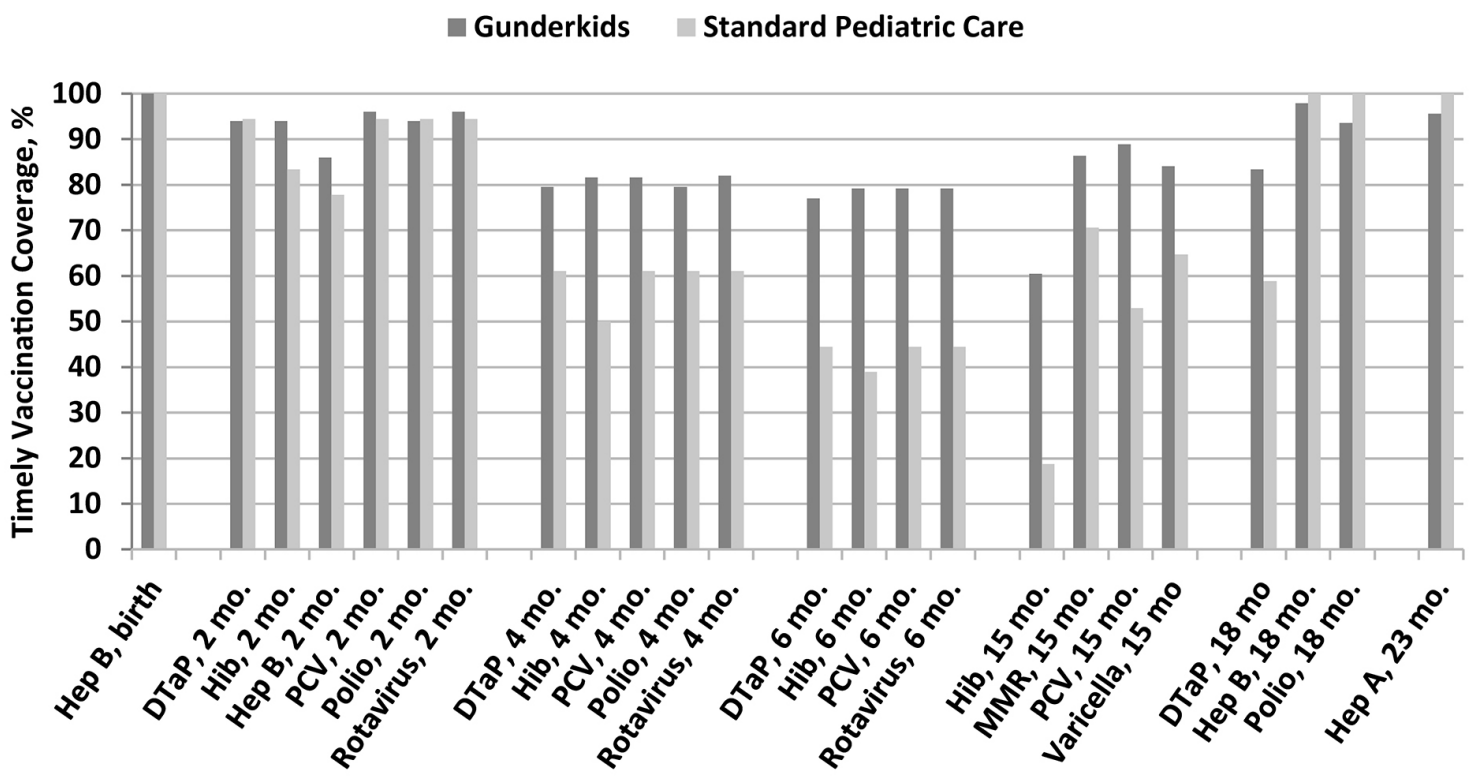

Figure 1. Comparison of timely vaccination coverage: GunderKids and Standard Pediatric Care groups by age. 
17 standard pediatric care infants who were eligible for the DTaP at 18 months, $10(58.8 \%)$ achieved vaccination coverage, compared with 35 (83.3\%) of the 42 eligible GunderKids infants.

Twenty-three months. First dose hepatitis A (Hep A) was the only vaccination due between 12 and 23 months. The standard pediatric group demonstrated slightly higher vaccination coverage than the GunderKids group (100\% vs 95.6\%); however, no significant differences were found between groups $(P=.99)$.

\section{Discussion}

This study showed higher percentages of timely vaccination coverage for GunderKids infants compared with those receiving standard pediatric care, with significant differences for all vaccinations at 6 months of age. However, results also showed a decreased trend for overall timely vaccination coverage from birth to 15 months, which has been evidenced in similar studies specifically addressing age-appropriate pediatric vaccinations over time. ${ }^{15,16}$ Literature also supports that parents are more likely to have their children vaccinated when clinicians integrate an honest non-confrontational manner in approaching childhood vaccinations ${ }^{10}$ and if provider competence is trusted. ${ }^{11}$

A systematic review of relational components of care delivery models focusing on harm reduction among perinatal women

Table 2. Timely Vaccination Coverage of Infants in GunderKids and Standard Pediatric Care Groups

\begin{tabular}{|c|c|c|c|c|c|}
\hline \multirow[b]{2}{*}{$\begin{array}{l}\text { Vaccination Type } \\
\text { by Age Due }\end{array}$} & \multicolumn{2}{|c|}{$\begin{array}{l}\text { Number received / } \\
\text { Number eligible (\%) }\end{array}$} & \multirow[b]{2}{*}{$P$} & \multirow[b]{2}{*}{ OR } & \multirow[b]{2}{*}{$\mathrm{Cl}$} \\
\hline & $\begin{array}{l}\text { GunderKids } \\
(n=50)\end{array}$ & $\begin{array}{c}\text { Standard Pediatric } \\
\text { Care }(n=20)\end{array}$ & & & \\
\hline \multicolumn{6}{|l|}{ Birth } \\
\hline Hep B & $50 / 50(100.0)$ & $20 / 20(100.0)$ & .99 & 1.1 & $0.2-5.5$ \\
\hline \multicolumn{6}{|l|}{2 Months } \\
\hline DTaP & $47 / 50(94.0)$ & $17 / 18(94.4)$ & .99 & 1.2 & $0.2-8.5$ \\
\hline Hib & $47 / 50(94.0)$ & 15/18 (83.3) & .33 & 3.1 & $0.6-15.0$ \\
\hline Hep B & $43 / 50(86.0)$ & $14 / 18(77.8)$ & .46 & 1.8 & $0.5-6.7$ \\
\hline PCV & $48 / 50(96.0)$ & $17 / 18(94.4)$ & .99 & 1.7 & $0.2-13.5$ \\
\hline Polio & $47 / 50(94.0)$ & $17 / 18(94.4)$ & .99 & 1.2 & $0.2-8.5$ \\
\hline Rotavirus & $48 / 50(96.0)$ & $17 / 18(94.4)$ & .99 & 1.7 & $0.2-13.5$ \\
\hline \multicolumn{6}{|l|}{4 Months } \\
\hline DTaP & $39 / 49(79.6)$ & $11 / 18(61.1)$ & .11 & 2.5 & $0.8-7.7$ \\
\hline $\mathrm{Hib}$ & 40/49 (81.6) & $9 / 18(50.0)$ & .01 & 4.3 & $1.4-13.4$ \\
\hline PCV & 40/49 (81.6) & $11 / 18(61.1)$ & .10 & 2.8 & $0.9-8.9$ \\
\hline Polio & 39/49 (79.6) & $11 / 18(61.1)$ & .20 & 2.5 & $0.8-7.7$ \\
\hline Rotavirus & $41 / 50(82.0)$ & $11 / 18(61.1)$ & .11 & 2.8 & $0.9-9.1$ \\
\hline \multicolumn{6}{|l|}{6 Months } \\
\hline DTaP & $37 / 48(77.1)$ & 8/18 (44.4) & .011 & 4.0 & $1.3-12.3$ \\
\hline $\mathrm{Hib}$ & 38/48 (79.2) & 7/18 (38.9) & .002 & 5.6 & $1.8-17.7$ \\
\hline PCV & $38 / 48$ (79.2) & $8 / 18$ (44.4) & .006 & 4.5 & $1.5-14.1$ \\
\hline Rotavirus & $38 / 48(79.2)$ & 8/18 (44.4) & .006 & 4.5 & $1.5-14.1$ \\
\hline \multicolumn{6}{|l|}{15 Months } \\
\hline Hib & $26 / 43(60.5)$ & $3 / 16$ (18.8) & .004 & 5.8 & $1.6-21.9$ \\
\hline MMR & 38/44 (86.4) & $12 / 17(70.6)$ & .26 & 2.6 & $0.7-9.6$ \\
\hline PCV & 40/45 (88.9) & 9/17 (52.9) & .004 & 6.6 & $1.8-23.8$ \\
\hline Varicella & $37 / 44(84.1)$ & $11 / 17(64.7)$ & .16 & 2.8 & $0.8-9.8$ \\
\hline \multicolumn{6}{|l|}{18 Months } \\
\hline DTaP & $35 / 42(83.3)$ & $10 / 17(58.8)$ & .08 & 3.4 & $1.0-11.5$ \\
\hline Hep B & 48/49 (98.0) & 18/18 (100) & .99 & 0.9 & $0.0-22.4$ \\
\hline Polio & $44 / 47$ (93.6) & $18 / 18(100)$ & .55 & 0.3 & $0.0-7.0$ \\
\hline \multicolumn{6}{|c|}{23 Months ( $1^{\text {st }}$ dose $)$} \\
\hline Hep A & $43 / 45(95.6)$ & $15 / 15(100)$ & .99 & 0.6 & $0.0-12.3$ \\
\hline
\end{tabular}

DTaP, diphtheria, tetanus, and pertussis; Hep A, hepatitis A; Hib, Haemophilus influenzae type b; Hep B, hepatitis B; MMR, measles, mumps, and rubella; PCV, pneumococcal vaccine; OR, odds ratio; $\mathrm{Cl}$, confidence interval 
with SUD, found that outcomes of programs employing comprehensive, integrated, interdisciplinary care are promising and are linked to caring relationships with health care providers. ${ }^{17}$ A review of four randomized clinical trials of parental SUD intervention programs and children's mental and behavioral outcomes found that programs focusing on improving parenting skills and family functioning may effectively reduce childhood challenges and enhance their psychosocial functioning, ${ }^{3}$ which is a goal of the GunderKids program. Results from this preliminary study and current evidence suggest that developing respectful caring relationships between health care providers and parents are integral to healthy outcomes in families affected by substance use disorders.

This study had several limitations. First, the sample size of both groups was small, with the average GunderKids' data showing significantly longer documented follow up time than standard pediatric care data. A larger sample size may contribute to more reliable statistical results. Second, the study was conducted within a regional population that is not ethnically or racially diverse. Third, the retrospective design using EHR data limited type and quality of data retrieval and analyses. Last, confounders may include voluntary enrollment in the GunderKids program that may represent mothers who have better access to this clinical service or are more motivated to participate in clinical activities focusing on their children's health. These limitations affect generalizability of results and direct applicability to clinical practice. Despite these limitations, the GunderKids group's predominantly higher percentage of timely vaccination coverage is noteworthy and calls for additional studies to evaluate the clinical effectiveness of programs like GunderKids.

\section{Conclusion}

GunderKids infants received more timely vaccinations than those who received standard pediatric care, suggesting that the program's focus on building relationships with mothers who have SUD has a positive association with the overall care their children receive. Results also suggest that timeliness of vaccination coverage might be used as a proxy for measuring program effectiveness. These results show promise that specialized programs like GunderKids may assist in mitigating short-term as well as long-term adverse health consequences among children exposed to familial SUD. The GunderKids program is fundamentally focused on care coordination and building provider and staff relationships with children and their families. Continued GunderKids data development, retrieval, and analysis of program metrics and outcomes will evolve longitudinally with additional focus on relationships between GunderKids program participants and health care providers. Findings suggest that integrating care of mothers who have SUD with the care of their infants may be associated with better childhood health outcomes than infants who receive standard pediatric care. Further IRBapproved GunderKids research will use the master dataset to retrospectively address factors contributing to outcomes of healthy child anthropometric measures, uses and types of clinical services, parental maintenance of child custody, and childhood maltreatment. Results support continued study to prospectively determine clinical, individual, and organizational factors influencing parental behaviors within SUD-exposed families that lead to healthy childhood outcomes.

\section{Acknowledgments}

The authors wish to acknowledge Dawn Steffes, Clinical Research Coordinator, for her database development, data collection, queries, and mentoring others in this work. Additionally, the suggestions, critique, and editorial expertise of Cathy L. Fischer, MA, ELS Scientific Writer and Editor, are greatly appreciated.

\section{References}

1. Feder KA, Mojtabai R, Musci RJ, Letourneau EJ. U.S. adults with opioid use disorder living with children: Treatment use and barriers to care. J Subst Abuse Treat. 2018;93:31-37.

2. Schauberger CW, Newbury EJ, Colburn JM, Al-Hamadani M. Prevalence of illicit drug use in pregnant women in a Wisconsin private practice setting. Am J Obstet Gynecol. 2014;211(3):255.e1-255.e2554. doi:10.1016/j. ajog.2014.03.023.

3. Calhoun S, Conner E, Miller M, Messina N. Improving the outcomes of children affected by parental substance abuse: a review of randomized controlled trials. Subst Abuse Rehabil. 2015;6:15-24.

4. Smith VC, Wilson CR; COMMITTEE ON SUBSTANCE USE AND PREVENTION. Families affected by parental substance use. Pediatrics. 2016;138(2):e20161575.

5. Galligan K, Comiskey CM. Hidden harms and the number of children whose parents misuse substances: a stepwise methodological framework for estimating prevalence. Subst Use Misuse. 2019;54(9):1429-1437.

6. Kuppens S, Moore SC, Gross V, Lowthian E, Siddaway AP. The enduring effects of parental alcohol, tobacco, and drug use on child well-being: a multilevel meta-analysis. Dev Psychopathol. 2020;32(2):765-778.

7. Callaghan T, Crimmins J, Schweitzer RD. Children of substance-using mothers: child health engagement and child protection outcomes. J Paediatr Child Health. 2011;47(4):223-227.

8. Budzak-Garza AE, Allmon Dixson AL, Holzer RA, Lillard-Pierce KE, Devine CJ. GunderKids: design of a clinical care management program for parents with substance abuse and their newborn children with a focus on preventing child abuse. WMJ. 2018;117(1):29-33. 
9. Niccols A, Milligan K, Smith A, Sword W, Thabane L, Henderson J. Integrated programs for mothers with substance abuse issues and their children: A systematic review of studies reporting on child outcomes. Child Abuse Negl. 2012;36(4):308-322.

10. Healy CM. Commentary on "Parental vaccinehesitancy: Understanding the problem and searching for a resolution". Hum Vaccin Immunother. 2014;10(9):2597-2599.

11. Benin AL, Wisler-Scher DJ, Colson E, Shapiro ED, Holmboe ES. Qualitative analysis of mothers' decisionmaking about vaccines for infants: the importance of trust. Pediatrics. 2006;117(5):1532-1541.

12. Luman ET, Barker LE, Shaw KM, McCauley MM, Buehler JW, Pickering LK. Timeliness of childhood vaccinations in the United States: days undervaccinated and number of vaccines delayed. JAMA.

2005;293(10):1204-1211.

13. Rodgers L, Shaw L, Strikas R, et al. Frequency and cost of vaccinations administered outside recommended ages - 2014 data from 6 sentinel sites of immunization information systems. J Pediatr. 2018;193:164-171.

14. Strikas RA; Centers for Disease Control and Prevention (CDC); Advisory Committee on Immunization Practices (ACIP); ACIP Child/Adolescent Immunization Work Group. Advisory committee on immunization practices recommended immunization schedules for persons aged 0 through 18 years-United States, 2015. MMWR Morb Mortal Wkly Rep. 2015;64(4):93-94.

15. Kurosky SK, Davis KL, Krishnarajah G. Completion and compliance of childhood vaccinations in the United States. Vaccine. 2016;34(3):387-394.

16. Walton S, Cortina-Borja M, Dezateux C, et al. Measuring the timeliness of childhood vaccinations: Using cohort data and routine health records to evaluate quality of immunisation services. Vaccine. 2017;35(51):7166-7173.

17. Kramlich D, Kronk R. Relational care for perinatal substance use: a systematic review. MCN Am J Matern Child Nurs. 2015;40(5):320-326.

\section{Author Affiliations}

Ann C. Falkenberg-Olson, PhD*; Karen L. Hayter, PhD*; Renee A. Holzer, BSN ; Jayne M. Laylan, MSN广; Andrew J. Borgert, PhD*; and Ann E. Budzak Garza, MD广

*Gundersen Health System-Gundersen Medical Foundation, La Crosse, WI

†Gundersen Health System, La Crosse, WI 